\title{
Disentangling the NIR/optical emission of the black hole XTE J1650-500 during outburst ${ }^{\star}$
}

\author{
P. A. Curran ${ }^{1}$, S. Chaty ${ }^{1,2}$, and J. A. Zurita Heras ${ }^{3}$ \\ ${ }^{1}$ Laboratoire AIM, UMR 7158 CEA/DSM-CNRS-Université Paris Diderot, Irfu/Service d'Astrophysique, \\ CEA-Saclay, 91191 Gif-sur-Yvette Cedex, France \\ e-mail: peter.curran@cea.fr \\ 2 Institut Universitaire de France, 103 Bd Saint-Michel, 75005 Paris, France \\ 3 François Arago Centre, APC, Université Paris Diderot, CNRS/IN2P3, CEA/DSM, Observatoire de Paris, 13 rue Watt, \\ 75205 Paris Cedex 13, France
}

Received 15 March 2012 / Accepted 27 September 2012

\section{ABSTRACT}

\begin{abstract}
Context. While the sources of X-ray and radio emission in the different states of low-mass X-ray binaries are relatively well understood, the origin of the near-infrared (NIR) and optical emission is more often debated. It is likely that the NIR/optical flux originates from an amalgam of different emission regions, because it occurs at the intersecting wavelengths of multiple processes.

Aims. We aim to identify the NIR/optical emission region(s) of one such low-mass X-ray binary and black hole candidate, XTE J1650-500, via photometric, timing, and spectral analyses.

Methods. We present unique NIR/optical images and spectra, obtained with the ESO-New Technology Telescope, during the peak of the 2001 outburst of XTE J1650-500.

Results. The data suggest that the NIR/optical flux is due to a combination of emission mechanisms including a significant contribution from X-ray reprocessing and, at early times in the hard state, a relativistic jet that is NIR/radio dim compared to similar sources. Conclusions. The jet of XTEJ1650-500 is relatively weak compared to that of other black hole low-mass X-ray binaries, possibly because we observe as it is being "turned off" or quenched at the state transition. While there are several outliers to the radio-X-ray correlation of the hard state of low-mass X-ray binaries, XTE J1650-500 is the first example of an outlier to the NIR/optical-X-ray correlation.
\end{abstract}

Key words. accretion, accretion disks - ISM: jets and outflows - infrared: stars - X-rays: individuals: XTE J1650-500 -

$\mathrm{X}$-rays: binaries

\section{Introduction}

Transient low-mass X-ray binaries (LMXBs) are, for most of the time, in a state of quiescence with faint or non-detected $\mathrm{X}$-ray emission and near-infrared (NIR)/optical emission dominated by the main-sequence companion star (possibly with significant contribution from the cold accretion disk). They are often only discovered when - powered by an increased level of accretion onto the central compact object (black hole or neutron star) - there is a dramatic increase of the X-ray, NIR/optical and radio flux. During these outbursts the systems are observed to go through several high-energy (X-ray) spectral states before returning to a quiescent state, usually on timescales of weeks, months, or even longer.

LMXBs with a black hole compact object are observed (in $\mathrm{X}$-rays) initially in a generally low-intensity, power-law dominated, hard state before transitioning to a usually higher intensity, thermal-dominant, soft state that decreases in flux and evolves via a late hard states back into a quiescent state. Some outbursts also display a steep power law state with a steeper spectral slope than the regular hard state during the transition between hard and soft states. In general, the X-ray hardness versus intensity diagrams (HIDs) are observed to follow canonical trajectories (e.g., Homan et al. 2001; Homan \& Belloni 2005;

* Based on observations made with the European Southern Observatory telescopes obtained from the ESO/ST-ECF Science Archive Facility.
Belloni 2010) and are often (though not always) indicative of the black hole nature of the compact object. Additionally, the hard states are associated with aperiodic variability of the light curve not present in the soft state and, in hard and steep power law states, quasi-periodic oscillations (QPOs) are detected (for a fuller description of the various possible states see McClintock \& Remillard 2006)

The origin of the NIR, optical and ultraviolet (UV) emission from a black hole LMXB is much less understood than the origins of the X-ray or radio emission because the optical wavelengths are at the intersection of a number of different emission mechanisms (for reviews of optical properties of LMXBs see e.g., van Paradijs \& McClintock 1995; Charles \& Coe 2006). The companion star is generally relatively dim and may not contribute significant flux during outburst when other mechanisms are active. Intrinsic, thermal emission from the hot, outer accretion disk may contribute at UV and optical wavelengths (e.g., Shakura \& Sunyaev 1973; Frank et al. 2002) though the reprocessing of X-rays in the same region of the accretion disk is thought to be a significant source of flux at wavelengths from UV through to NIR (e.g., Cunningham 1976; van Paradijs \& McClintock 1994). Recently, evidence has been mounting that the relativistic jet, usually detected in radio, also produces a significant contribution to the NIR flux, at least in the hard state (e.g., Jain et al. 2001; Corbel \& Fender 2002; Chaty et al. 2003; Russell et al. 2006), and it is possible that the power law X-ray emission in the hard state extends to, and contributes at, optical 
Table 1. Nights of observations.

\begin{tabular}{lcccc}
\hline \hline Night & MJD & Filters & Size $^{\dagger}\left({ }^{\prime}\right)$ & ESO ID \\
\hline $2001-09-08$ & 52161 & $J, H, K_{\mathrm{S}}$ & $5 \times 5$ & $67 . \mathrm{B}-0486$ \\
& & $G B F, G R F^{*}$ & - & $67 . \mathrm{B}-0486$ \\
$2001-09-14$ & 52167 & $V, R, I$ & $9 \times 9$ & $67 . \mathrm{B}-0315$ \\
$2001-09-15$ & 52168 & $R I L D \# 1^{*}$ & - & $67 . \mathrm{B}-0486$ \\
$2001-09-24$ & 52177 & $J, H, K_{\mathrm{S}}$ & $4 \times 4$ & $67 . \mathrm{D}-0200$ \\
$2001-10-04$ & 52187 & $J, H, K_{\mathrm{S}}$ & $5 \times 5$ & $68 . \mathrm{D}-0316$ \\
$2001-10-06$ & 52189 & $K_{\mathrm{S}}$ & $4 \times 4$ & $68 . \mathrm{D}-0316$ \\
$2001-10-09$ & 52192 & $V, R, I$ & $9 \times 9$ & $68 . \mathrm{A}-0440$ \\
$2001-10-10$ & 52193 & $R I L D \# 1^{*}$ & - & $68 . \mathrm{D}-0144$ \\
$2001-10-13$ & 52196 & $V$ & $1 \times 1$ & $68 . \mathrm{D}-0144$ \\
$2003-05-13$ & 52772 & $I$ & $3 \times 10$ & $71 . \mathrm{D}-0337$ \\
\hline
\end{tabular}

Notes. ${ }^{(\dagger)}$ Final image size after cropping; ${ }^{(*)}$ Spectra.

wavelengths. This emission is ascribed to the inner regions of the system - either a corona, advection-dominated accretion flow, or the base of a compact jet - which we will refer to as the corona for simplicity.

The black hole X-ray transient XTEJ1650-500 was initially detected by the Rossi X-ray Timing Explorer (RXTE) on September 5, 2001 (Remillard 2001). In the following days, its variable nature was confirmed (Remillard 2001; Markwardt et al. 2001; Wijnands et al. 2001) and quasi-periodic oscillations (QPOs) indicative of black hole LMXBs were observed (Revnivtsev \& Sunyaev 2001). Optical (Castro-Tirado et al. 2001; Augusteijn et al. 2001; Buxton \& Londish 2001) and ATCA radio (Groot et al. 2001) counterparts were also reported at the time. The canonical black hole state behaviour of the source over the $\sim 100$ day long outburst is well documented in both X-ray (Rossi et al. 2004) and radio (Corbel et al. 2004), while evidence of the black hole nature of the compact object is offered by the confirmation of QPOs (Homan et al. 2003), the detection of a relativistic Fe $\mathrm{K} \alpha$ line (e.g., Miller et al. 2002; Miniutti et al. 2004) and a derived mass of $\simeq 5.1 M_{\odot}$ (Slaný \& Stuchlík 2008).

The optical observations of the source during outburst (cited above) were limited to a few epochs, but on June 10, 2002, in quiescence, Sanchez-Fernandez et al. (2002) obtained 15 optical spectra with FORS2 on the $8.2 \mathrm{~m}$ VLT at ESO, Paranal. Using these spectra and relative optical photometry (obtained with the $6.5 \mathrm{~m}$ Clay telescope at Las Campanas Observatory, Atacama during May, June, and August 2002), Orosz et al. (2004) derive an orbital period of 0.32 days ( 7.69 h). On August 2, 2002 Garcia $\&$ Wilkes (2002) estimated the quiescent optical magnitude. In this paper we present the only significant NIR/optical observations during outburst, obtained by the ESO NTT in September and October 2001 (Table 1), which comprises all available unpublished, archived ESO data of the source. In Sect. 2 we introduce the observations and reduction methods, while in Sect. 3 we present the results of our photometric, timing, and spectral analyses of the data. We discuss the interpretation of our findings within the context of black hole LMXBs in Sect. 4 and summarise in Sect. 5.

\section{Observations and reduction}

\subsection{Photometry}

Optical $(V, R, I)$ and NIR $\left(J, H, K_{\mathrm{S}}\right)$ data were obtained with the ESO Multi-Mode Instrument (EMMI; Dekker et al. 1986) and the Son of ISAAC (SofI) infrared spectrograph and imaging
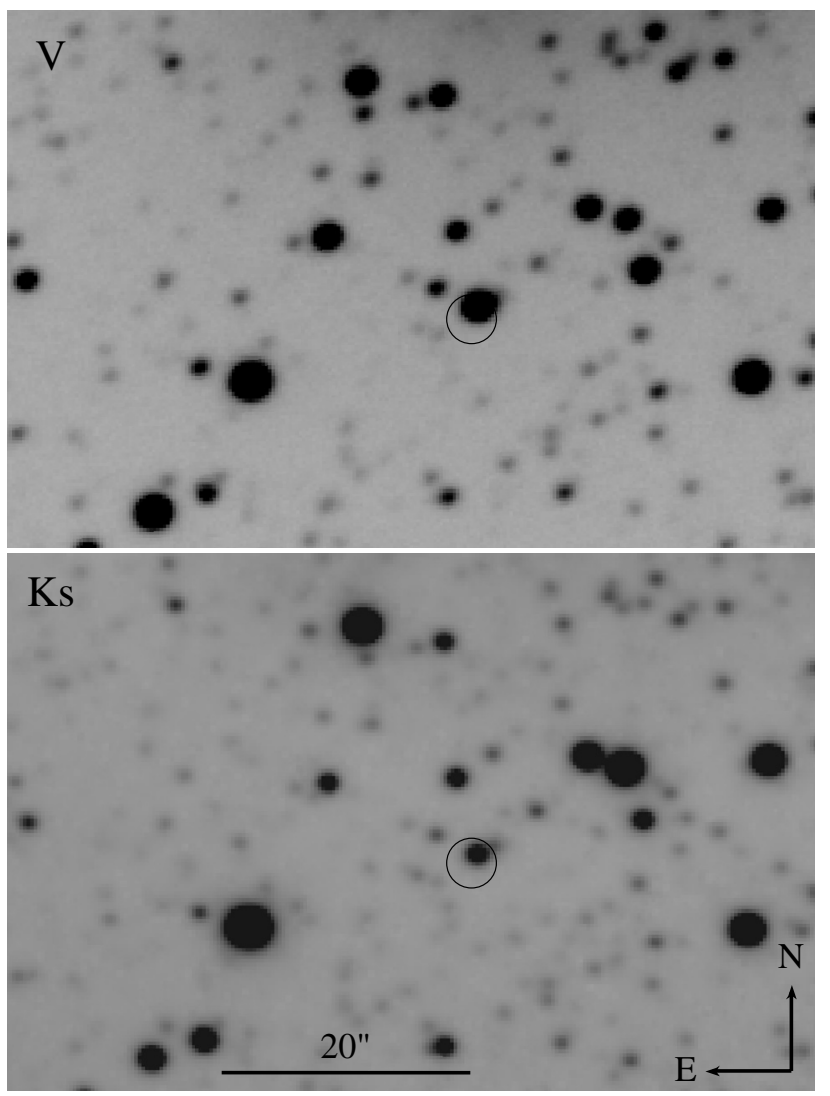

Fig. 1. NTT $60^{\prime \prime} \times 40^{\prime \prime}$ finding charts (upper: 970 s $V$ image; lower: $910 \mathrm{~s} K_{\mathrm{S}}$ image) with the $2^{\prime \prime}$ optical positional uncertainty (CastroTirado et al. 2001) marked by a circle.

camera on the $3.58 \mathrm{~m}$ ESO-New Technology Telescope (NTT), respectively, on a number of nights in September and October 2001, and in May 2003 (Table 1). Data were reduced using the IRAF package, in which crosstalk correction, bias-subtraction, flatfielding, sky subtraction, bad pixel correction and frame addition were carried out as necessary. NIR data obtained on the nights of September 8 and October 4 did not use a dithered pointing pattern as is usual for NIR images, therefore it was not possible to produce a reliable sky for subtraction; instead the sky produced on September 24 was used. This should be of minimum effect because we used relative photometry to estimate magnitudes.

The images were astrometrically calibrated against 2MASS (Skrutskie et al. 2006) or USNO-B1.0 (Monet et al. 2003) within the GAIA package. The position of the source was derived via the point spread function (PSF) photometry of the deep $K_{\mathrm{S}}$ band image on October 6 (MJD 52188 ; seeing $\approx 1.2^{\prime \prime}$ ) as 16:50:00.95 $-49: 57: 44.34$ with a positional error ${ }^{1}$ dominated by the $0.1^{\prime \prime}$ 2MASS systematic uncertainty (Fig. 1).

Relative PSF photometry was carried out on the final images using the DAOPHOT package (Stetson 1987) within IRAF. However, the optical data obtained on September 14 and October 9 were so poorly binned that producing an acceptable PSF model was impossible (full width at half maximum, $F W H M \lesssim 2$ pixels); hence these images use relative aperture photometry. The NIR magnitudes (Table 2; Fig. 2) were calibrated against the 2 MASS catalogue using $\approx 200$ objects per image, after outliers were removed. The calibration, at least for the $K_{\mathrm{S}}$ band, was confirmed on the October $6 K_{\mathrm{S}}$ image and

1 All uncertainties in this paper are given with a confidence of $1 \sigma$. 
Table 2. Optical and NIR exposures and magnitudes.

\begin{tabular}{cccc}
\hline \hline MJD-50000 & Filter & $N \times \operatorname{Exp}(\mathrm{s})$ & Magnitude \\
\hline 2167.00354 & $V$ & $1 \times 30$ & $17.1 \pm 0.2$ \\
2191.99546 & $V$ & $1 \times 120$ & $17.3 \pm 0.3$ \\
2195.99182 & $V$ & $97 \times 10$ & $17.4 \pm 0.4$ \\
2166.99704 & $R$ & $1 \times 150$ & $17.0 \pm 0.4$ \\
2167.00545 & $R$ & $1 \times 30$ & $16.7 \pm 0.4$ \\
2191.99848 & $R$ & $1 \times 120$ & $17.0 \pm 0.4$ \\
2192.00531 & $R$ & $1 \times 40$ & $17.3 \pm 0.4$ \\
2167.00002 & $I$ & $1 \times 60$ & $15.86 \pm 0.13$ \\
2167.00699 & $I$ & $1 \times 30$ & $15.69 \pm 0.13$ \\
2192.00104 & $I$ & $1 \times 90$ & $16.3 \pm 0.3$ \\
2192.00696 & $I$ & $1 \times 40$ & $16.0 \pm 0.2$ \\
2772.17920 & $I$ & $4 \times 300$ & $>19.0$ \\
2161.02124 & $J$ & $5 \times 20$ & $14.42 \pm 0.11$ \\
2176.98651 & $J$ & $9 \times 10$ & $14.65 \pm 0.09$ \\
2186.98230 & $J$ & $5 \times 20$ & $14.79 \pm 0.10$ \\
2161.02907 & $H$ & $5 \times 20$ & $13.79 \pm 0.11$ \\
2176.99397 & $H$ & $9 \times 10$ & $14.16 \pm 0.12$ \\
2186.99013 & $H$ & $5 \times 20$ & $14.12 \pm 0.16$ \\
2161.03691 & $K_{\mathrm{S}}$ & $5 \times 20$ & $13.29 \pm 0.13$ \\
2177.00145 & $K_{\mathrm{S}}$ & $9 \times 10$ & $13.82 \pm 0.12$ \\
2186.99797 & $K_{\mathrm{S}}$ & $5 \times 20$ & $13.99 \pm 0.16$ \\
2188.97400 & $K_{\mathrm{S}}$ & $130 \times 7$ & $14.18 \pm 0.13$ \\
\hline 2160.98738 & $G B F$ & $2 \times 180$ & - \\
2160.99906 & $G R F$ & $2 \times 360$ & - \\
2167.99490 & $R I L D \# 1$ & $2 \times 600$ & - \\
2193.01287 & $R I L D \# 1$ & $2 \times 900$ & - \\
\hline & & & \\
\hline
\end{tabular}

four Persson et al. (1998) photometric standards, observed on the same night (the only night on which reliable NIR standards were available).

Optical magnitudes (Table 2; Fig. 2) were calculated relative to $\approx 10$ relatively isolated field stars, which were calibrated on the September 14 images against Landolt (1992) photometric standards, observed on the same night (the only night on which optical standards were obtained). The errors on the optical magnitudes are dominated by this absolute photometric calibration but unfortunately, due to the saturation of USNO-B1.0 objects in the field, we are unable to calibrate against that, or other, catalogues. The upper limit of the late $I$ band image is approximated from the dimmest observable object in the region of interest. Because we were only able to obtain aperture photometry on September 14 and October 9, the measured magnitude is likely to suffer contamination from the unresolved, nearby sources (see Orosz et al. 2004, for a better resolved image from the $6.5 \mathrm{~m}$ Clay telescope). To correct for this, we estimated the magnitude of the contaminating sources to be $I=19.2 \pm 0.5$ from the magnitude at the position in the late $I$ band image (May 13, 2003) when the source had faded; $V=18.4 \pm 0.4$ from the residual of the PSF photometry on October 13,$2001 ; R=18.7 \pm 0.4$ from an interpolation of the $I$ and $V$ contaminating magnitudes. The difference in magnitude ranges from $\gtrsim 1 \mathrm{mag}$ for the $V$-band to $\gtrsim 3 \mathrm{mag}$ for the $I$ band. In each case, given the relatively large absolute calibration error, the corrected magnitude (Table 2 ) is consistent, within errors, with the magnitude uncorrected for contamination.

The data obtained on October 6 and October 13 consisted of multiple high-cadence, "fast" photometry images, in $K_{\mathrm{S}}$ and $V$-bands, respectively, to investigate possible short-term variability (Table 2) up to $\sim 2-3 \mathrm{~h}$, with an approximate sampling of an image every $90 \mathrm{~s}$. PSF photometry was carried out on each of these individual images, again using DAOPHOT. Source magnitudes were calculated relative to a number of field stars (10 in

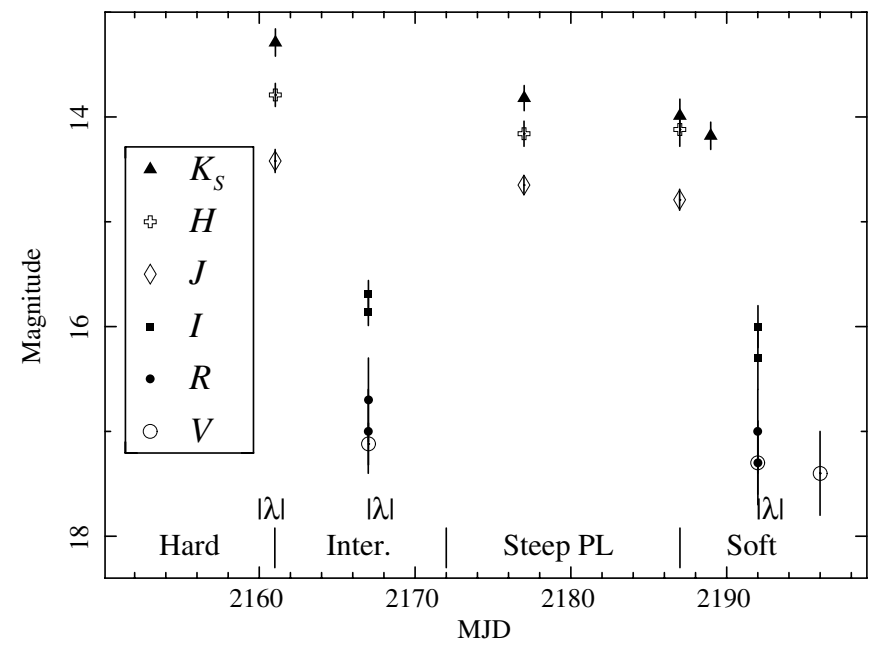

Fig. 2. $V, R, I, J, H$, and $K_{\mathrm{S}}$ band light curves (as presented in Table 2) with the periods of the hard, intermediate, steep power law and soft states (Corbel et al. 2004) marked. $|\lambda|$ marks the epochs of the spectral observations.

NIR and 8 in optical) and normalized so that the weighted average is equal to zero. In each, a few magnitudes were eliminated due to unsatisfactory PSF subtraction residuals which became obvious during visual inspection of the images.

\subsection{Spectral energy distributions}

The observed magnitudes (Table 2) or, if there were multiple observations per band per night, the weighted average magnitudes, were first converted into flux densities, $F_{v}$, at frequency $v$. They were then converted into flux per filter, $F_{\text {filter }}$ in units of photons $\mathrm{cm}^{-2} \mathrm{~s}^{-1}$. This conversion was made via $F_{\text {filter }}=$ $1509.18896 F_{v}(\Delta \lambda / \lambda)$ where $\lambda$ and $\Delta \lambda$ are the effective wavelength and width of the filter in question. XSPEC compatible files for spectral energy distribution (SED) fitting were then produced from the flux per filter value using the FTOOL, flx $2 x s p$. In Fig. 3 we correct for the Galactic extinction in the direction of the source (Schlegel et al. 1998) of $E_{B-V}=0.923\left(A_{K} \sim 0.3\right.$, $A_{V} \sim 2.9$; Cardelli et al. 1989) $)^{2}$. This value of extinction is consistent with that implied (Güver \& Özel 2009) from the X-ray absorption of this source (Montanari et al. 2009), even though the source is likely closer (Homan et al. 2006) and should not suffer the full effect of Galactic dust.

\subsection{Spectroscopy}

Optical and NIR spectra were obtained with EMMI and SofI on nights in September and October 2001 (Tables 1, 2). EMMI obtained red (3850-10000 ̊) low-dispersion spectra using grism\#1 (RILD 1) on two nights. SofI obtained usable blue $(G B F ; 9500-16400 \AA)$ and red $(G R F ; 15300-25200 \AA)$ lowresolution spectra on a single night, while spectra from another two nights (September 18 and October 4, 2001) were not useful due to a very high level of spatial dispersion, as well as a lack of spectra for wavelength calibration on those nights. The data were reduced using the IRAF package in which crosstalk correction, flatfielding and dark- or bias subtraction were carried out as necessary. To correct for NIR sky, the dithered NIR exposures were

2 This extinction should be treated with caution as estimates so close to the Galactic plane $(<5 \mathrm{deg})$ are uncertain. 
A\&A 547, A41 (2012)

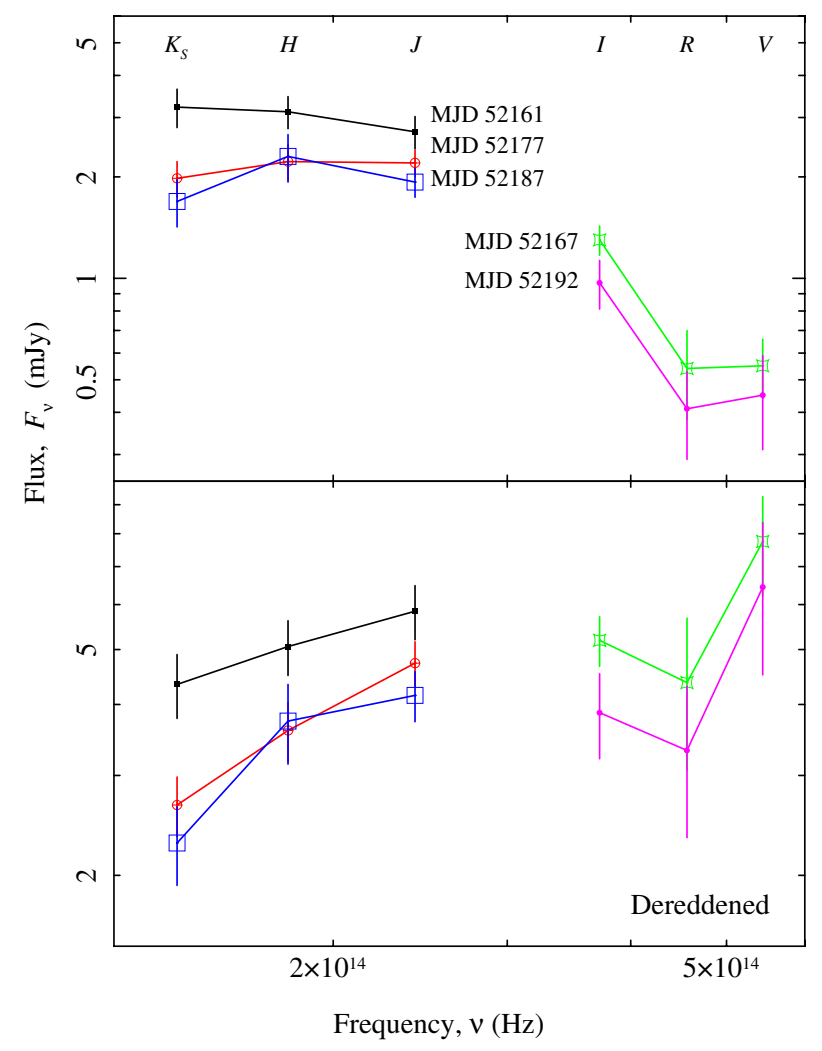

Fig. 3. Flux versus frequency plot at five different epochs, uncorrected (upper) and corrected (lower) for the Galactic extinction in the direction of the source of $E_{B-V}=0.923$.

subtracted from each other and the resulting spectra summed later.

Spectra were extracted and reduced within the IRAF package, noao. twodspec. Wavelength calibrations were performed against helium + argon (optical) or xenon (NIR) lamps whose spectra were extracted using the same parameters as for the relevant source. Atmospheric telluric features significantly affect the spectra and were corrected for by dividing the source spectrum by that of a telluric standard at a similar airmass, using the telluric tool within IRAF. This procedure often causes artefacts in the corrected spectra and, in the case of our optical spectra, these artefacts are significant, which is why we did not apply this procedure. Neither the optical nor the NIR spectra were flux calibrated, but they were normalized.

The NIR spectra (from the night of September 8 2001) were combined with the optical spectrum from the night of September 15,2001 and the final spectrum, from $3850-25200 \AA$, is plotted in Fig. 4. Owing to discrepancies in the optical wavelength calibrations between the two nights, we are unable to sum the two optical spectra and instead plot the spectra with the lower noise. The (wavelength-dependent) resolution of the final spectrum is $7-10 \AA$, with a wavelength calibration error of $\$ 20 \AA$ (optical) or $\lesssim 40 \AA$ (NIR). The apparent features just redward of $4000 \AA$ and blueward of $10^{4} \AA$ are at the edge of the optical grism range where the response is not reliable. The high levels of noise at $\sim 14000 \AA$ and $\sim 19000 \AA$ are artefacts of the correction for telluric features, while the general trend towards higher noise at the red end is a real effect of the observations. An absorption feature with a FWHM of $60 \AA$ at $7625 \AA$ is detected in the optical spectrum of the night of September 15, but this is an atmospheric feature, also detected with similar properties in the spectroscopic standard on that night. This feature is not detected in the October 10 spectrum, though this spectrum has a root mean square noise about four times greater than the September 15 spectrum.

\section{Results}

\subsection{High-cadence photometry}

Though the high-cadence light curves (Figs. 5 and 6), particularly the $K_{\mathrm{S}}$ band, seem to display some level of variability by eye, this is not supported by a careful analysis of the data. The standard deviation of the $V$ data is $0.012 \mathrm{mag}$, compared to an average error on the data points of $0.011 \pm 0.002$. We fitted a constant value to the light curve which, for the $V$-band, returns $\chi_{v}^{2}=143.1 /(97-1)=1.49$, statistically inconsistent with being an acceptable fit at $>99 \%$ confidence. However, if we apply the same tests to a number of nearby sources of similar magnitude (and hence similar signal-to-noise ratio) we find similar standard deviations $(0.011-0.014)$ and $\chi_{v}^{2}(1.31-2.05)$, for the same number of degrees of freedom. This implies that any variability is not intrinsic to the source itself but is likely noise. Likewise, the $K_{\mathrm{S}}$ band data, which have a standard deviation of 0.059 mag compared to an average error on the data points of $0.017 \pm 0.007$, returns $\chi_{v}^{2}=1222 /(128-1)=9.6$, inconsistent with a nonvariable source. However,several nearby sources of similar magnitude also return similar standard deviations (0.056-0.086) and $\chi_{v}^{2}(9.1-14.9)$, again implying that any variability is not intrinsic to the source itself and that, for the $K_{\mathrm{S}}$ band, the errors on the individual photometric points are underestimated, likely due to e.g. the difficulties of sky subtraction and PSF modelling in such a crowded field (Fig. 1).

Furthermore, we used the IRAF task, pdm - an implementation of the phase dispersion minimization method of Stellingwerf (1978) - to test for periodicity in the variability of the light curves (see periodograms in lower panels of Figs. 5 and 6). We find no reliable periodicity in either of the observed bands as the tests returned Stellingwerf statistics, $\Theta \approx 1$ for all periods less than the length of observations and broad minima of $\Theta \gtrsim 0.7$ for longer test periods. While the $\Theta$ statistic in each band was generally lower than that for the nearby comparison objects (introduced above, dashed lines in periodograms), there was no strong local minimum that would sugest a periodic variability. Neither is there any sign of periodicity when the $\Theta$ statistic of the source is normalized relative to that of the comparison objects. This null result was also confirmed using the Lomb-Scargle method for unevenly sampled data (Press \& Rybicki 1989), which shows no significant peak in power over the period ranges in question.

We also tested both light curves to see if either were consistent with a sinusoidal periodicity similar to that of Orosz et al. (2004), who measured a quiescent period of 0.3205 days (7.69 $\mathrm{h}$, compared to the $\sim 2-3 \mathrm{~h}$ duration of our observations) and an $R$ band peak-to-peak amplitude of $0.2 \mathrm{mag}$. This fit to the $K_{\mathrm{S}}$ light curve returns $\chi_{v}^{2}=7.2$ (125 degrees of freedom) while similar fits to the comparison objects return $\chi_{v}^{2}$ of between 10.3 and 14.2. A similar fit to the $V$-band light curve of the source returns $\chi_{v}^{2}=1.5$ (93 degrees of freedom) while fits to the comparison objects return $\chi_{v}^{2}$ of between 1.5 and 3.4. While it would seem that both bands could be consistent with this period, it is necessary in both cases that the epochs of observations fall serendipitously at the minima of the periodic function, which seems unlikely. Indeed, to observe such a period in outburst would be physically unrealistic since the periodicity was detected through orbital eclipsing; during outburst, where the optical emission is 5 mag brighter than quiescence $(V \sim 24$, 
P. A. Curran et al.: Disentangling the NIR/optical emission of the black hole XTE J1650-500 during outburst

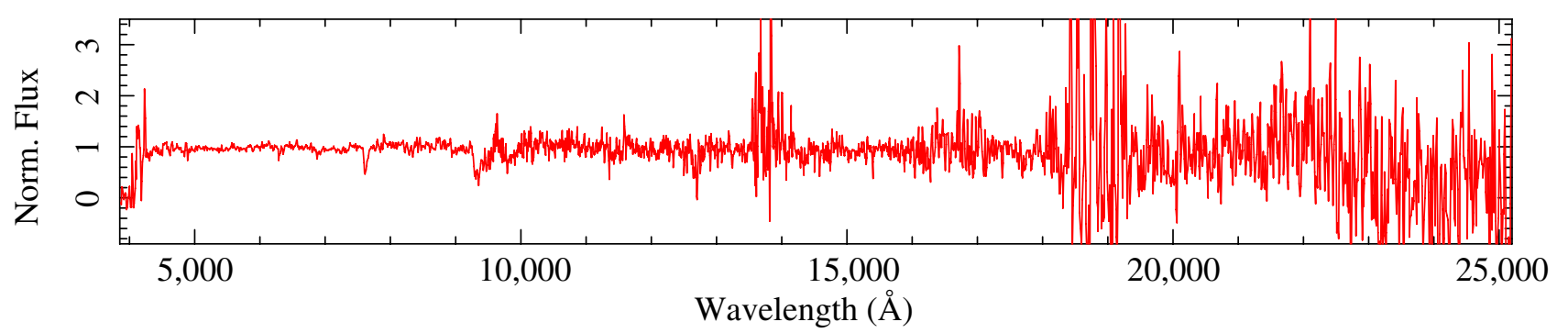

Fig. 4. Final normalized spectrum from 3850 to $25200 \AA$. The apparent features just redward of $4000 \AA$ and blueward of $10000 \AA$ are at the edge of the optical grism range where the response is not reliable. The high levels of noise at $\sim 14000 \AA$ and $\sim 19000 \AA$ are artefacts of the telluric correction while the absorption feature at $7625 \AA$ is atmospheric.
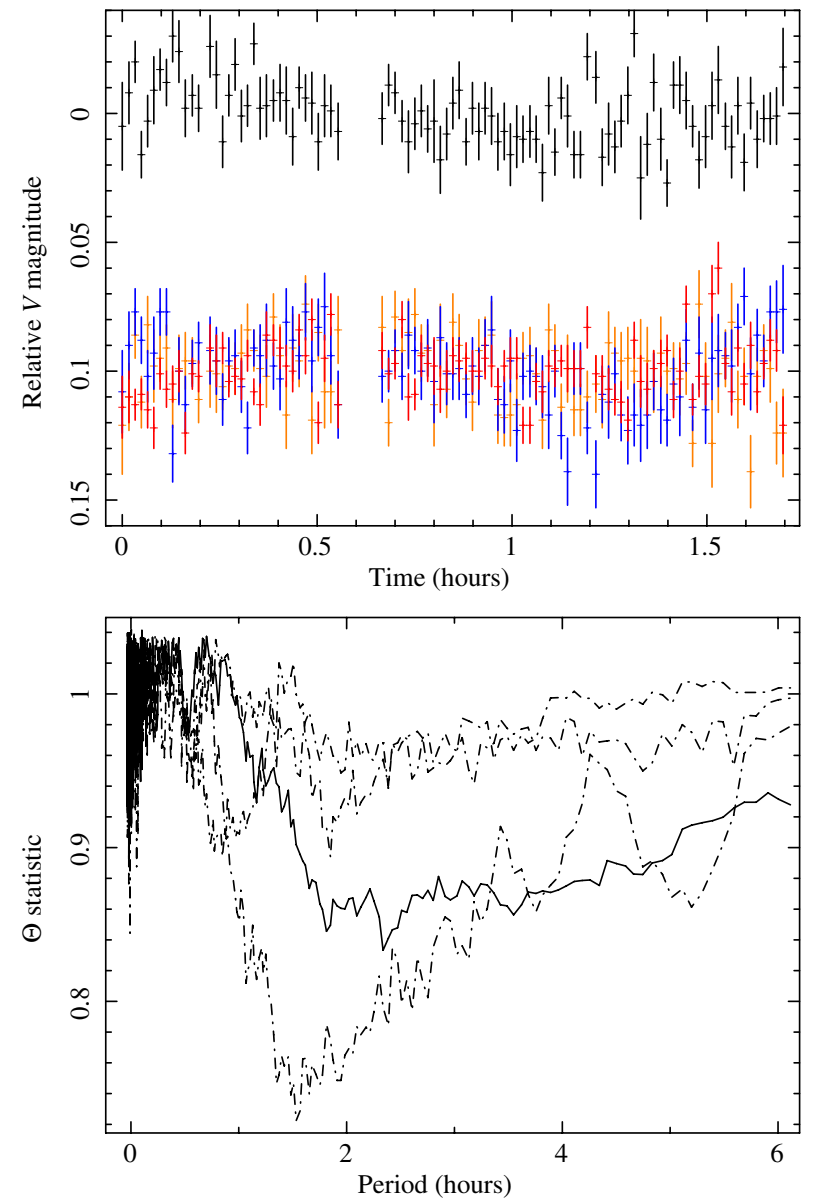

Fig. 5. Top: high-cadence $V$-band light curve of the source along with three comparison objects of a similar magnitude (normalized to a relative magnitude of 0.1$)$ and, bottom, the periodogram $(\Theta$ statistic versus period) for the source (solid line) and the nearby comparison objects (dashed lines).

$R \sim 22$; Garcia \& Wilkes 2002), this would not be detected. However, so-called "superhump" modulations, indicative of the orbital period, have been observed in the hard state of several X-ray binaries (e.g., Zurita et al. 2008).

\subsection{Spectral energy distributions}

Though the light curves (Fig. 2) and flux-versus-frequency plots (Fig. 3) display apparent hints of NIR colour evolution as well as a possible $V$-band excess, the spectral indices ( $\alpha$, where $\left.F_{v} \propto v^{\alpha}\right)$ of the two sets of $V, R, I$ data and the three sets of $J, H, K_{\mathrm{S}}$ data are all consistent within the (not particularly
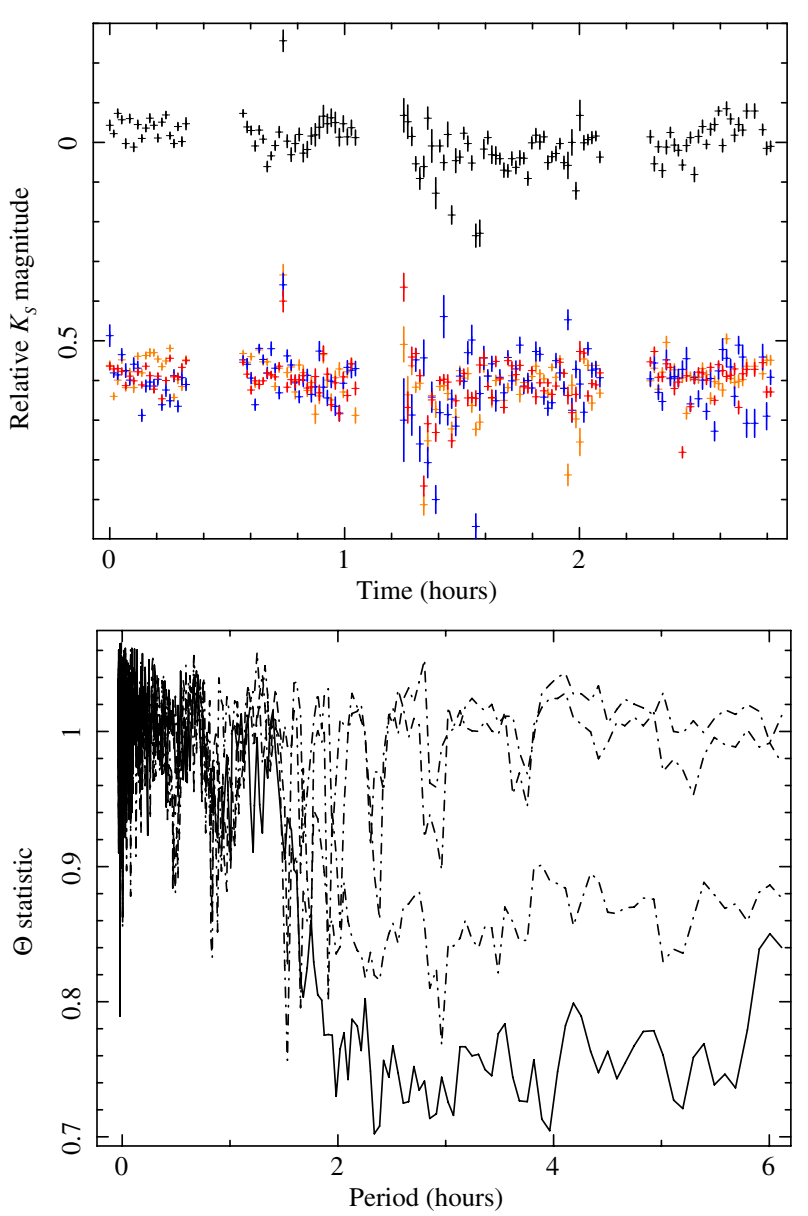

Fig. 6. Top: high-cadence $K_{\mathrm{S}}$ band light curve of the source along with three comparison objects of a similar magnitude (normalised to a relative magnitude of 0.6$)$ and, bottom, the periodogram $(\Theta$ statistic versus period) for the source (solid line) and the nearby comparison objects (dashed lines).

well constrained) errors. Furthermore, a simultaneous fit of all five (non-simultaneous) SED epochs does not support significant spectral evolution, as the data are well fit $\left(\chi_{v}^{2}=0.72\right.$ for 8 degrees of freedom) by an absorbed power law of spectral index, $\alpha=0.8 \pm 0.2$, with the extinction set to $E_{B-V}=0.923$, the Galactic value in that direction (Schlegel et al. 1998). The only evolution, at least given the quality of the data, is that of the flux offset, which decreases in time, as expected from the light curves. It is important to note here that while spectral evolution is not statistically supported, neither is it ruled out as a possibility. 


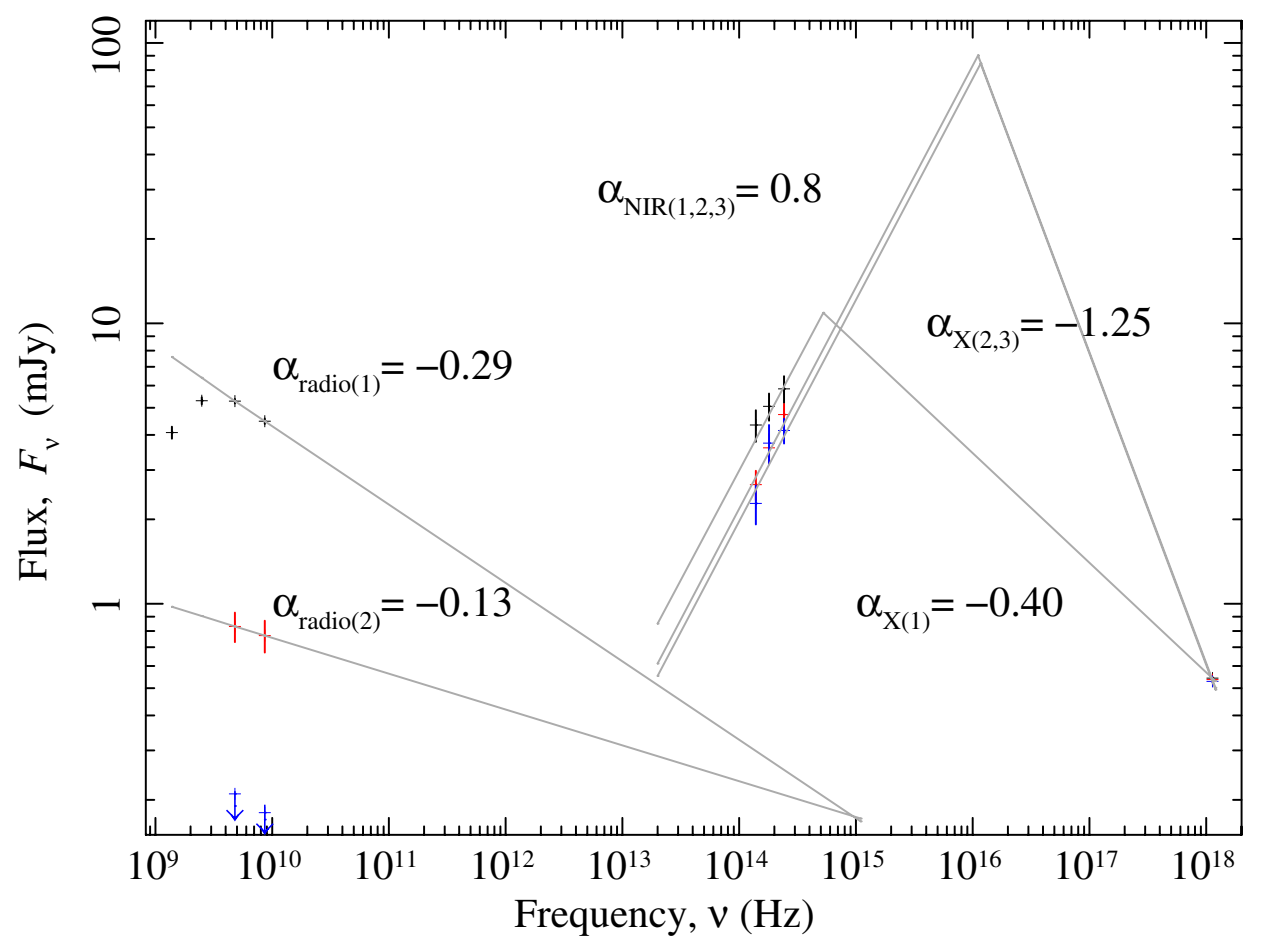

Fig. 7. Dereddened/unabsorbed (except for radio) flux-versus-frequency plot at three epochs (1 = MJD 52 161, black; 2 = MJD 52 177, red; $3=$ MJD 52 187, blue). Spectral indices, $\alpha$, are from previously published sources, except for the NIR. Power laws are purely phenomenological in order to show the relative behavior at different frequencies.

Alternatively, the combined SEDs could also be acceptably fit by simple black body radiation at any temperature $\gtrsim 3100 \mathrm{~K}\left(\gtrsim 2.7 \times 10^{-4} \mathrm{keV}\right)$ with extinctions, $E_{B-V} \lesssim 1.6$, or at $7000 \mathrm{~K}\left(6 \times 10^{-4} \mathrm{keV}\right)$ for the Galactic extinction $\left(\chi_{v}^{2}=\right.$ 1.67). Additionally, the SEDs are also consistent with black body radiation from an accretion disk of temperature $\gtrsim 5500 \mathrm{~K}$ $\left(\gtrsim 4.7 \times 10^{-4} \mathrm{keV}\right)$ with extinctions, $E_{B-V} \lesssim 0.8$, or at $\approx 6 \times 10^{6} \mathrm{~K}$ $(0.5 \mathrm{keV})$ for the Galactic extinction $\left(\chi_{v}^{2}=1.86\right)$. It is hence clear that the SEDs are not particularly constraining and results should be treated with caution, especially because of the time-averaged nature of the above fits.

\subsection{Spectroscopy}

The final spectrum (Fig. 4) displays a single possible feature that we might attribute to the source: a tenuous line (with a peak at $\sim 6$ times the noise and a FWHM of $10 \AA$ ) is detected at $16700 \AA$. The same feature is visible in the spectrum before telluric correction, therefore is not an artefact of that process. This line could correspond to an HI line at $16810 \AA$ (Brackett series, $\eta$ ), though given the difference in wavelength and the very narrow FWHM compared to the spectral resolution (7-10 $\mathrm{A})$, it is likely that this line is not real. If this line were real we would also expect to detect other lower energy Brackett series lines that would have higher abundances, but we do not detect any, again suggesting that the line may be a statistical fluctuation. Augusteijn et al. (2001), in their optical spectrum obtained on September 8, 2001 (on the night of our NIR spectra and seven nights prior to our low-noise optical spectrum) identified Balmer series emission lines, but we observe no such lines with a signal-to-noise ratio $\gtrsim 4$. Neither do we detect the broad, double-peaked $\mathrm{H} \alpha$ emission line from the accretion disk or the weak absorption lines from the companion star identified by Sanchez-Fernandez et al. (2002) in their VLT spectra from June 10, 2002 (i.e., in quiescence).

\section{Discussion}

The states of XTEJ1650-500 are defined, from X-ray and radio observations, as being hard up to MJD $~ 52161$; hard/intermediate up to MJD 52 172; steep power law up to MJD 52 187; thermal-dominant/soft up to MJD 52 232; and hard after that date (Corbel et al. 2004). Our observations were hence obtained in the very late, initial hard/intermediate, steep power law and in the first few days of the soft state (Fig. 2). Specifically, our multi-band NIR observations were obtained within $5 \mathrm{~h}$ of observations 2, 3, and 4 of Corbel et al., in the hard and steep power law states. In Fig. 7 the dereddened NIR and radio data are plotted along with the X-ray flux from that paper (converted to Jansky at $4.7 \mathrm{keV}$ ). The common spectral index of the NIR observations (Sect. 3.2) is also plotted along with the fitted spectral indices of the radio (Corbel et al. 2004, who used a thermal free-free absorbed power law) and the X-ray (Rossi et al. 2004) at those epochs. One can see that epoch 1, in the hard state, is characterised by a high level of radio emission and a relatively shallow-power-law dominated X-ray flux, while epochs 2 and 3 display declining radio flux and steep power-lawdominated X-ray emission. One of the most obvious aspects of this plot is that while the radio flux drops by over an order of magnitude, the X-ray flux remains relatively stable, dropping only fractionally. As pointed out by Corbel et al., this is consistent with the radio originating from a self-absorbed compact jet while also indicating that the jet contributes little, if any, flux to the X-ray emission. Furthermore, the decrease to background of radio flux is consistent with the assumed behaviour of the jet in a black hole system that is quenched during the soft, thermal state (Corbel et al. 2004; Fender et al. 2004).

\subsection{The source of NIR/optical emission}

It is expected that the NIR/optical emission of a black hole LMXB originates from a combination of the companion star, the 
relativistic jet, intrinsic thermal or reprocessed X-rays from the accretion disk, and a possible contribution from the corona (see Sect. 1). The estimated quiescent optical magnitudes of this system $(V \sim 24, R \sim 22$ Garcia \& Wilkes 2002; $I>19.0$ herein) are significantly $(\sim 100-500$ times) dimmer than those observed in outburst and hence imply that the companion star has no measurable effect on the emission during outburst. If the observed NIR flux were due exclusively to the jet, we would expect an almost flat spectral index $(\alpha \approx 0)$, much shallower than that observed $(\alpha=0.8 \pm 0.2)$. We would also expect an approximately linear relationship between the NIR and radio flux (which is assumed to be, and is consistent with being, due only to jet emission). However, it is unclear how this relationship would evolve over a state transition, as is being observed here, since flux at one frequency may decrease earlier or faster than that at another frequency. We find that the NIR flux falls off much more gradually with $F_{\text {NIR }} \propto F_{\text {Radio }}^{0.21 \pm 0.07}$ (at both radio frequencies), and maintains a high level of emission even as the jet/radio emission goes to zero in the soft state. Additionally, over the same time, the X-ray flux falls off even less steeply than the NIR $\left(F_{\mathrm{NIR}} \propto F_{\mathrm{X}}^{25 \pm 10}\right)$, therefore it seems plausible that at least some of the NIR emission originates from the jet while the remaining emission must be due to the accretion disk, reprocessing and/or coronal emission.

Unfortunately the X-ray flux cannot be extrapolated to imply an accretion disk contribution to the NIR, as the accretion disk is not generally observed to make a significant contribution to the X-ray in the hard or steep power law states. We would expect to observe spectral emission lines if the NIR emission originated from an accretion disk, but we observe none in our spectra during the hard/intermediate states; however, this is not to say that the accretion disk is not making a, possibly significant, contribution at these times. Neither can we extrapolate the $\mathrm{X}$-ray flux to imply the corona's contribution because, while it is clear that the power law contribution in the hard and steep power law states must break at low energies, we cannot constrain the form of this break. Since the significant spectral change of the X-ray does not seem to affect the NIR spectra, which remain constant, one might be tempted to imply that the corona does not contribute to the NIR, but because of the poorly constrained nature of the SED and the lack of knowledge of the low-energy spectral shape of the corona, this is not necessarily the case. As mentioned earlier (Sect. 1), black hole LMXBs display significant X-ray variability in the hard state at frequencies from $\sim 10^{-3}$ to $10^{4} \mathrm{~Hz}$, which is absent from the soft state, and this hard state variability has also been observed in the optical from $\sim 10^{-4}$ to $10 \mathrm{~Hz}$ (e.g., Casella et al. 2010; Chaty et al. 2011). The high level of variability in both optical and X-ray is often attributed to the non-thermal emission of the jet or corona. We find no such variability (Sect. 3.1) in our high-cadence optical and NIR observations (spanning $\sim 10^{-4}$ to $10^{-1} \mathrm{~Hz}$ ), which might be indicative of emission associated with these regions, consistent with the source's soft state at those epochs.

If the NIR emission was primarily due to X-ray reprocessing, the observed NIR flux should be proportional to the observed $\mathrm{X}$-ray flux as $\propto F_{\mathrm{X}}^{\sim 0.5}$ (van Paradijs \& McClintock 1994; or for more detailed calculations, Coriat et al. 2009) but instead it follows the much steeper relationship of $F_{\text {NIR }} \propto F_{\mathrm{X}}^{25 \pm 10}$. However, it must be noted here that the level of reprocessing (and the NIR $\mathrm{X}$-ray relationship) is very sensitive to the $\mathrm{X}$-ray spectral shape, and hence the X-ray range sampled by a particular instrument. Over a state transition, as is being observed here, the X-ray spectrum is undergoing dramatic changes of spectral shape; consequently, while the X-ray flux over the measured range remains constant, the flux over the range contributing to the reprocessing may be significantly variable. We do find that the absolute optical magnitude of the source $\left(M_{V}=2.2\right)$ assuming a distance of $2.6 \pm 0.7 \mathrm{kpc}$ (Homan et al. 2006), agrees well with the observed correlation with $\Sigma=\left(L_{\mathrm{X}} / L_{\mathrm{Edd}}\right)^{1 / 2} P^{2 / 3}=0.4$ (van Paradijs \& McClintock 1994; Deutsch et al. 2000), when we use the observed period, $P=7.69$ days (Orosz et al. 2004), and the derived mass of $\simeq 5.1 M_{\odot}$ (Slaný \& Stuchlík 2008). This correlation was derived for reprocessing, implying that the optical flux may originate from this process. Furthermore, the lack of observed variability in the long term optical - in contrast to the NIR - light curves (Fig. 2) is consistent with the $F_{\text {Optical }} \propto F_{\mathrm{X}}^{0.5}$ relationship expected from reprocessing. The possible, though not statistically significant, $V$-band excess (Fig. 3) may be due to reprocessing peaking in that, or a bluer, band.

\subsection{The weak jet of XTE J1650-500}

It has been noted (Corbel et al. 2004; Fender et al. 2010) that XTE J1650-500 is X-ray loud, relative to the radio, when compared to other black hole candidates (e.g., Corbel et al. 2000, 2003; Gallo et al. 2003; Fender et al. 2010; Coriat et al. 2011). The source also appears to be X-ray loud relative to the NIR, as in both the hard (epoch 1) and the steep power law (epochs 2, 3) states an extrapolation of the X-ray spectra overestimates - significantly in the steep power law state - the NIR flux, requiring a low-energy break of the X-ray spectrum. An excess X-ray emission is also suggested by a comparison of the NIR and $\mathrm{X}$-ray luminosities: assuming a distance of $2.6 \pm 0.7 \mathrm{kpc}$ (Homan et al. 2006), we find NIR and X-ray luminosities in the hard state (epoch 1) of $L_{\mathrm{NIR}} \approx 10^{33} \mathrm{erg} \mathrm{s}^{-1}$ and $L_{\mathrm{X}} \approx 10^{37} \mathrm{erg} \mathrm{s}^{-1}$ (each with a $40 \%$ error, dominated by the uncertainty on distance). These luminosities fall under the LMXB (black hole) hard state correlation of Russell et al. (2006), implying that either the source is X-ray loud (by $\gtrsim 3$ orders of magnitude) or that the source is NIR dim (by $\gtrsim 2$ orders of magnitude). For these luminosities to fall on the correlation, the distance would have to be a factor of 30 , or more, greater $(\sim 80 \mathrm{kpc})$, which is of course unphysical compared to the size of the Galaxy ( $\sim 35 \mathrm{kpc}$ diameter); indeed, considering the source's position in the Galactic plane $\left(l, b=336.7^{\circ}-3.4^{\circ}\right)$ any distance much greater than $8.5 \mathrm{kpc}$ is unlikely. In the soft state (which has luminosities similar to those of the hard state) the source appears to be NIR and optically dim relative to the distribution of other soft state LMXBs (Russell et al.), but not significantly so. Furthermore, as noted by Gallo et al. (2008), the late-time quiescent X-ray luminosity $\left(L_{\mathrm{X}} \approx 3 \times 10^{30} \mathrm{erg} \mathrm{s}^{-1}\right)$ is at the lower end of the distribution of quiescent luminosities for black hole binaries, though, when compared with the quiescent optical luminosity $\left(L_{\text {Optical }} \approx 10^{30} \mathrm{erg} \mathrm{s}^{-1}\right)$, the X-ray luminosity falls on the correlation of Russell et al., albeit at the low end. Obviously, because of NIR/optical observational bias (i.e., we only observe the brightest sources), caution has to be exercised when implying that a source is of a low luminosity compared to a distribution as we do here for the soft and quiescent states. However, this observational bias should not affect our comparison to the hard state correlation. Given that the hard state X-ray luminosity of this source is very similar to the range of luminosities presented in Russell et al. $\left(10^{35}-10^{38} \mathrm{erg} \mathrm{s}^{-1}\right)$, while the NIR luminosity is at the low end of the observed range (at the quiescent level of other LMXBs), it seems more likely that the source is NIR, and hence radio, dim (at least quantitatively) as opposed to X-ray loud, as suggested by Corbel et al. (2004). 
While both the NIR and radio fall below their respective hard state correlations with the X-ray (in fact, this is the first example of an outlier to the NIR/optical correlation), the calculated NIR luminosities are more consistent with the correlation for the hard states of neutron star LMXBs (Russell et al. 2006) - in contrast to the canonical black hole behaviour of the observed $\mathrm{X}$-ray properties of this source (Sect. 1). Likewise, the radio luminosities of the source fall on the efficient branch of the radioX-ray correlation and are similar to the luminosities of neutron stars (Coriat et al. 2011). The accretion flow in the hard state of black hole binaries is generally radiatively inefficient and as such a significant amount of the gravitational potential may be converted to relativistic jet flow (i.e., kinematically efficient; e.g., Gallo et al. 2005), although that energy may also pass unobserved across the black hole's event horizon, advected onto the compact object (Narayan et al. 1996). Conversely, the accretion flow in neutron star binaries (and the soft state of black holes) is radiatively efficient and thus thermal accretion emission is a more effective method of energy radiation than a jet (i.e., kinematically inefficient, see e.g., Fender et al. 2003). Hence, it is likely that the detected jet of XTEJ1650-500 is weak due to radiatively efficient accretion (this is what Coriat et al. refer to, qualitatively, as X-ray loud). This is also consistent with the radio (Corbel et al. 2004) and X-ray (Rossi et al. 2004; Montanari et al. 2009; Corbel et al. 2004) light curves, which peak (except at very soft energies, where the soft state thermal component is dominant) by the time of our first observation, and it is consistent with the X-ray definitions of state since our first observation is at the very end of the initial hard state. Thus what we observe in both radio and NIR is a weak jet that may be in the process of being "turned off" or quenched due to the state transition from hard to intermediate as the accretion flow transitions from geometrically thick, optically thin and radiatively inefficient (but kinematically efficient) to geometrically thin, optically thick and radiatively efficient.

\section{Conclusions}

The data of XTE J1650-500 suggest that the jet contributes to the NIR flux, at least at early times, before being quenched completely by the time of the later, steep power law state observation. However, we find that the NIR flux falls off much more gradually than the radio and maintains a high level of emission even as the jet/radio emission goes to zero - suggesting that there is also significant contribution from X-ray reprocessing and possibly the accretion disk and/or corona, though we cannot distinguish which of these might dominate due to a poorly constrained SED and a lack of indicative features of either, such as spectral lines or photometric variability.

The observed radio and NIR luminosities during the hard state are orders of magnitude lower than expected for a source of this X-ray luminosity, and while several outliers to the radioX-ray correlation of the hard state of LMXBs exist, this is the first such example of an outlier to the NIR/optical-X-ray correlation. The NIR/radio dim emission suggests that the jet of XTE J1650-500 is much weaker than in the hard states of other black hole LMXBs, possibly because we observe the jet as it is being "turned off", or quenched, at the state transition.

Acknowledgements. We thank S. Corbel for useful discussions on the manuscript and the anonymous referee for constructive comments. This work was supported by the Centre National d'Etudes Spatiales (CNES) and is based on observations obtained with MINE: the Multi-wavelength INTEGRAL NEtwork.
This research has made use of NASA's Astrophysics Data System and the SIMBAD database, operated at CDS, Strasbourg, France.

\section{References}

Augusteijn, T., Coe, M., \& Groot, P. 2001, IAU Circ., 7710, 2

Belloni, T. 2010, in The Jet Paradigm, Lecture Notes in Physics (Berlin: Springer Verlag), 794

Buxton, M. M., \& Londish, D. 2001, IAU Circ., 7715, 3

Cardelli, J. A., Clayton, G. C., \& Mathis, J. S. 1989, ApJ, 345, 245

Casella, P., Maccarone, T. J., O’Brien, K., et al. 2010, MNRAS, 404, L21

Castro-Tirado, A. J., Kilmartin, P., Gilmore, A., et al. 2001, IAU Circ., 7707, 3

Charles, P. A., \& Coe, M. J. 2006, in Optical, ultraviolet and infrared observations of X-ray binaries, eds. W. H. G. Lewin, \& M. van der Klis, 215

Chaty, S., Haswell, C. A., Malzac, J., et al. 2003, MNRAS, 346, 689

Chaty, S., Dubus, G., \& Raichoor, A. 2011, A\&A, 529, A3

Corbel, S., \& Fender, R. P. 2002, ApJ, 573, L35

Corbel, S., Fender, R. P., Tzioumis, A. K., et al. 2000, A\&A, 359, 251

Corbel, S., Nowak, M. A., Fender, R. P., Tzioumis, A. K., \& Markoff, S. 2003, A\&A, 400, 1007

Corbel, S., Fender, R. P., Tomsick, J. A., Tzioumis, A. K., \& Tingay, S. 2004, ApJ, 617, 1272

Coriat, M., Corbel, S., Buxton, M. M., et al. 2009, MNRAS, 400, 123

Coriat, M., Corbel, S., Prat, L., et al. 2011, MNRAS, 414, 677

Cunningham, C. 1976, ApJ, 208, 534

Dekker, H., Delabre, B., \& Dodorico, S. 1986, in SPIE Conf. Ser. 627, ed. D. L. Crawford, 339

Deutsch, E. W., Margon, B., \& Anderson, S. F. 2000, ApJ, 530, L21

Fender, R. P., Gallo, E., \& Jonker, P. G. 2003, MNRAS, 343, L99

Fender, R. P., Belloni, T. M., \& Gallo, E. 2004, MNRAS, 355, 1105

Fender, R. P., Gallo, E., \& Russell, D. 2010, MNRAS, 406, 1425

Frank, J., King, A., \& Raine, D. J. 2002, Accretion Power in Astrophysics, third edition (Cambridge, UK: Cambridge University Press)

Gallo, E., Fender, R. P., \& Pooley, G. G. 2003, MNRAS, 344, 60

Gallo, E., Fender, R., Kaiser, C., et al. 2005, Nature, 436, 819

Gallo, E., Homan, J., Jonker, P. G., \& Tomsick, J. A. 2008, ApJ, 683, L51

Garcia, M. R., \& Wilkes, B. J. 2002, The Astronomer's Telegram, 104, 1

Groot, P., Tingay, S., Udalski, A., \& Miller, J. 2001, IAU Circ., 7708, 4

Güver, T., \& Özel, F. 2009, MNRAS, 400, 2050

Homan, J., \& Belloni, T. 2005, Ap\&SS, 300, 107

Homan, J., Wijnands, R., van der Klis, M., et al. 2001, ApJS, 132, 377

Homan, J., Klein-Wolt, M., Rossi, S., et al. 2003, ApJ, 586, 1262

Homan, J., Wijnands, R., Kong, A., et al. 2006, MNRAS, 366, 235

Jain, R. K., Bailyn, C. D., Orosz, J. A., McClintock, J. E., \& Remillard, R. A. 2001, ApJ, 554, L181

Landolt, A. U. 1992, AJ, 104, 340

Markwardt, C., Swank, J., \& Smith, E. 2001, IAU Circ., 7707, 2

McClintock, J. E., \& Remillard, R. A. 2006, in Black hole binaries, eds. W. H. G. Lewin, \& M. van der Klis, 157

Miller, J., Wijnands, R., Wojdowski, P., et al. 2002, The Astronomer's Telegram, 81,1

Miniutti, G., Fabian, A. C., \& Miller, J. M. 2004, MNRAS, 351, 466

Monet, D. G., Levine, S. E., Canzian, B., et al. 2003, AJ, 125, 984

Montanari, E., Titarchuk, L., \& Frontera, F. 2009, ApJ, 692, 1597

Narayan, R., McClintock, J. E., \& Yi, I. 1996, ApJ, 457, 821

Orosz, J. A., McClintock, J. E., Remillard, R. A., \& Corbel, S. 2004, ApJ, 616, 376

Persson, S. E., Murphy, D. C., Krzeminski, W., Roth, M., \& Rieke, M. J. 1998 , AJ, 116, 2475

Press, W. H., \& Rybicki, G. B. 1989, ApJ, 338, 277

Remillard, R. 2001, IAU Circ., 7707, 1

Revnivtsev, M., \& Sunyaev, R. 2001, IAU Circ., 7715, 1

Rossi, S., Homan, J., Miller, J. M., \& Belloni, T. 2004, Nucl. Phys. B Proc. Suppl., 132, 416

Russell, D. M., Fender, R. P., Hynes, R. I., et al. 2006, MNRAS, 371, 1334

Sanchez-Fernandez, C., Zurita, C., Casares, J., et al. 2002, IAU Circ., 7989, 1

Schlegel, D. J., Finkbeiner, D. P., \& Davis, M. 1998, ApJ, 500, 525

Shakura, N. I., \& Sunyaev, R. A. 1973, A\&A, 24, 337

Skrutskie, M. F., Cutri, R. M., Stiening, R., et al. 2006, AJ, 131, 1163

Slaný, P., \& Stuchlík, Z. 2008, A\&A, 492, 319

Stellingwerf, R. F. 1978, ApJ, 224, 953

Stetson, P. B. 1987, PASP, 99, 191

van Paradijs, J., \& McClintock, J. E. 1994, A\&A, 290, 133

van Paradijs, J., \& McClintock, J. E. 1995, in X-ray Binaries, eds.

W. H. G. Lewin, J. van Paradijs, \& E. P. J. van den Heuvel, 58

Wijnands, R., Miller, J. M., \& Lewin, W. H. G. 2001, IAU Circ., 7715, 2

Zurita, C., Durant, M., Torres, M. A. P., et al. 2008, ApJ, 681, 1458 